\title{
Electrospun Highly Porous Poly(L-lactic acid)-Dopamine- SiO2 Fibrous Membrane for Bone Regeneration
}

DOI:

10.1016/j.msec.2020.111359

\section{Document Version}

Accepted author manuscript

Link to publication record in Manchester Research Explorer

\section{Citation for published version (APA):}

Lu, Z., Wang, W., Zhang, J., Da Silva Bartolo, P. J., Gong, H., \& Li, J. (2020). Electrospun Highly Porous Poly(Llactic acid)-Dopamine- SiO2 Fibrous Membrane for Bone Regeneration. Materials Science and Engineering C: Materials for Biological Applications , 117. https://doi.org/10.1016/j.msec.2020.111359

\section{Published in:}

Materials Science and Engineering C: Materials for Biological Applications

\section{Citing this paper}

Please note that where the full-text provided on Manchester Research Explorer is the Author Accepted Manuscript or Proof version this may differ from the final Published version. If citing, it is advised that you check and use the publisher's definitive version.

\section{General rights}

Copyright and moral rights for the publications made accessible in the Research Explorer are retained by the authors and/or other copyright owners and it is a condition of accessing publications that users recognise and abide by the legal requirements associated with these rights.

\section{Takedown policy}

If you believe that this document breaches copyright please refer to the University of Manchester's Takedown Procedures [http://man.ac.uk/04Y6Bo] or contact uml.scholarlycommunications@manchester.ac.uk providing relevant details, so we can investigate your claim.

\section{OPEN ACCESS}




\title{
Electrospun Highly Porous Poly(L-lactic acid)-Dopamine-
}

\section{$\mathrm{SiO}_{2}$ Fibrous Membrane for Bone Regeneration}

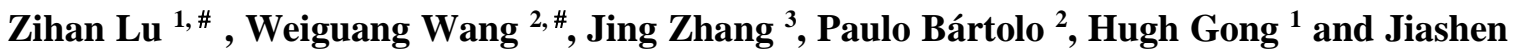 \\ $\mathbf{L i}^{1, *}$
}

${ }^{1}$ Department of Materials, School of Natural Sciences, Faculty of Science and Engineering, The University of Manchester, Manchester, M13 9PL, UK.

${ }^{2}$ Department of Mechanical, Aerospace and Civil Engineering, School of Engineering, Faculty of Science and Engineering, The University of Manchester, Manchester, M13 9PL, UK.

${ }^{3}$ School of Textile Science and Engineering, Xi'an Polytechnic University, Xi'an, ShaanXi, China, 710048

\# Co-first authors, contributed equally to this work.

* Corresponding authors. E-mail address: jiashen.li@ manchester.ac.uk

\begin{abstract}
Electrospinning has been widely used to fabricate polymer fibrous scaffolds for bone tissue engineering because of their highly porous structures. In order to improve the biocompatibility of polymer scaffolds, some nano particles have been introduced into electrospun fibres. For example, silica nanoparticles (SiNPs), with high surface area and good biocompatibility, have been used for bone tissue engineering for better bone cell attachment. In this work, porous poly(L-lactic acid) (PLLA) fibrous membrane with high surface area was fabricated by electrospinning and posttreatment process. The membrane can serve as substrates of SiNPs for bone tissue engineering. Dopamine (DOP) was applied to modify the surface of PLLA fibres, which improved the coating strength of SiNPs on PLLA fibres. SiNP coating significantly improved the mechanical properties
\end{abstract}


and hydrophilicity of PLLA/DOP/SiNP composite membranes. As a result of SiNPs coating, PLLA/DOP/SiNP membrane exhibited better cellular biocompatibility, more cell attachment and proliferation. These results demonstrate that porous PLLA/DOP/SiNP composite membrane with high surface area has high potential for periosteum in the field of bone regeneration applications.

Keywords: Dopamine (DOP) grafting; Electrospinning; Poly(L-lactic acid); Silica nanoparticle; Bone regeneration

\section{Introduction}

Bone defects are a challenging clinical problem which can be caused by many situations, such as trauma, infections or sarcoma resection [1-4]. In general, bone defects caused by trauma are often accompanied by periosteal damage. Periosteum is consisted of fibrous layer and osteogenic layer [5-7]. Usually, the fibrous layer is responsible for providing nourishment to cells via its capillary network and whilst original cells attached on the bone surface will continue to differentiate $[8,9]$. Periosteum plays a significate role in bone tissue engineering, it not only acts as a capillary network to supply nutrition, but also affects the cell differentiation. However, fabrication of an appropriate periosteum is still a challenge.

Electrospinning is a promising method to fabricate biomimetic membranes in the field of bone regeneration [10-13]. Membranes built by this technology have a native extracellular matrix like physical structure, which makes membranes more similar to damaged tissue structure [14-16]. Additionally, membranes produced through electrospinning have high surface area [17]. In comparison with other smooth surfaces, porous connected nanofibres can improve cell adhesion and proliferation $[18,19]$. In our previous publications, a two-step strategy has been reported to fabricate poly(L-lactic acid) (PLLA) fibrous membranes with high surface area $\left(75.60 \pm 0.60 \mathrm{~m}^{2} / \mathrm{g}\right)$ $[20,21]$. After PLLA nano/micro fibres were electrospun and collected, they were immersed in acetone for a few minutes at room temperature. As a result of the solvent-induced re-crystallisation of polymer chains, PLLA fibres were changed from solid to porous. With high surface area, these porous PLLA fibres and fibrous products have a wide range of potential applications in tissue 
engineering field. However, the hydrophobic nature of PLLA limits its biomedical properties. Therefore, it is very necessary to decorate or coat PLLA with some cell-affinity materials, such as proteins, peptides, keratin, hydroxyapatite, and so on. With these biomaterial coating, the cell adhesion and differentiation on PLLA fibres will certainly be promoted [22-25].

Bioactive polymer nanocomposites with amorphous silica nanoparticles (SiNPs) have been built as implants or scaffolds for bone regeneration [26, 27]. SiNP addition not only significantly enhances the mechanical properties and osteoblasts biocompatibility of various polymer materials, but also improves bone formation in vivo [28]. As mentioned before, periosteum can deliver oxygen/nutrition and has cell differentiation ability. As amorphous SiNPs have high surface area, high loading capacity and biocompatibility, they are a promising drug carrier [29, 30]. Researchers have successfully fabricated multifunctional bioactive polymer membranes containing SiNPs. For example, Chan et al. developed some inorganic nanoparticle $\left(\mathrm{SiO}_{2}, \mathrm{TiO}_{2}, \mathrm{ZrO}\right)$ loaded PLLA composite films via electrospinning for tissue engineering [31]. Antonio et al. fabricated poly $(\varepsilon-$ caprolactone)/SiNPs composite membranes to guide bone regeneration [15]. Although these results indicate that the addition of SiNPs can significantly improve the mechanical strength and osteoblasts cell differentiation of biomaterials, it is difficult to control the aggregation of SiNPs inside polymer matrix and their interaction with polymers [32, 33].

Coating nano particles on polymer scaffold can maximally use their biocompatibility. However, the adhesion strength between nano particles and polymer materials is a key issue for cell attachment and proliferation. Dopamine (DOP) is reported to have good biocompatibility and strong interactions with various materials to form adhesion layer through chemical reactions [3438]. Moreover, DOP is also verified to have reactivity with damaged tissues and good cell affinity [39]. For example, Wu et al. developed silk fibroin (SF)/DOP/E7 composite fibres via DOP grafting for bone regeneration. Their results show that DOP coating is a simple and useful method to graft short E7 peptides onto SF fibre surface with better hydrophilicity, cell attachment and proliferation [40]. 
In the present study, porous PLLA fibrous membranes were firstly produced by electrospinning and acetone treatment. Then SiNPs were synthesized and coated on the surface of PLLA fibres. DOP on PLLA fibres provided active functional groups which improved the adhesion strength of SiNPs to the porous membranes. The modified porous PLLA fibrous membranes with SiNP coating showed better biocompatibility which would promote cell attachment and proliferation. Meanwhile, these composite membranes also maintained higher mechanical properties and wettability.

\section{Materials and methods}

\subsection{Materials}

Poly (L-lactic) acid (PLLA, PL 65) was obtained from PURAC biochem, The Netherlands. Dichloromethane (DCM, 99.99\%), dopamine hydrochloride $(189.64 \mathrm{~g} / \mathrm{mol})$ and tris(hydroxymethyl)aminomethane (Tris) were purchased from Sigma-Aldrich (UK). Dimethyl Formamide (DMF, 99.80\%), tetraethyl orthosilicate (TEOS, 99.00\%) and ammonia hydroxide $\left(\mathrm{NH}_{3} \cdot \mathrm{H}_{2} \mathrm{O}, 35.00 \%\right)$ were obtained from Fisher Scientific Ltd (USA). Ethanol (99.97\%) and acetone $(99.70 \%)$ were purchased from VWR Science Co., Ltd (USA). Deionized (DI) water was obtained using USF-ELGA water purifier (USA). All reagents were applied as received without any further purification.

\subsection{Electrospinning of porous PLLA fibrous membrane}

The PLLA fibrous membrane was fabricated by electrospinning. A solution was prepared via dissolving PLLA (1.6 wt\%) in DCM and DMF mixture overnight. The ratio of DCM and DMF was 95:5 in weight. Afterwards, the blend solution was fed into a $30 \mathrm{~mL}$ syringe (Terumo Syringe, Japan) and pushed by a pump (TONGLI Tech Ltd., China) with $5 \mathrm{~mL} / \mathrm{h}$ feed rate. The process was executed with a $30 \mathrm{~cm}$ distance between nozzle tip and roll collector and under $23 \mathrm{kV}$ voltage. After the electrospun fibrous membrane was completely dried, it was cut and removed from the collector. Then a piece of PLLA membrane was immersed in acetone for $5 \mathrm{~min}$ at room temperature. 
After acetone treatment, the membrane was dried in a fume cupboard for $24 \mathrm{~h}$. The post process induces PLLA self-recrystallization which causes porous structure, as shown in Figure 1.

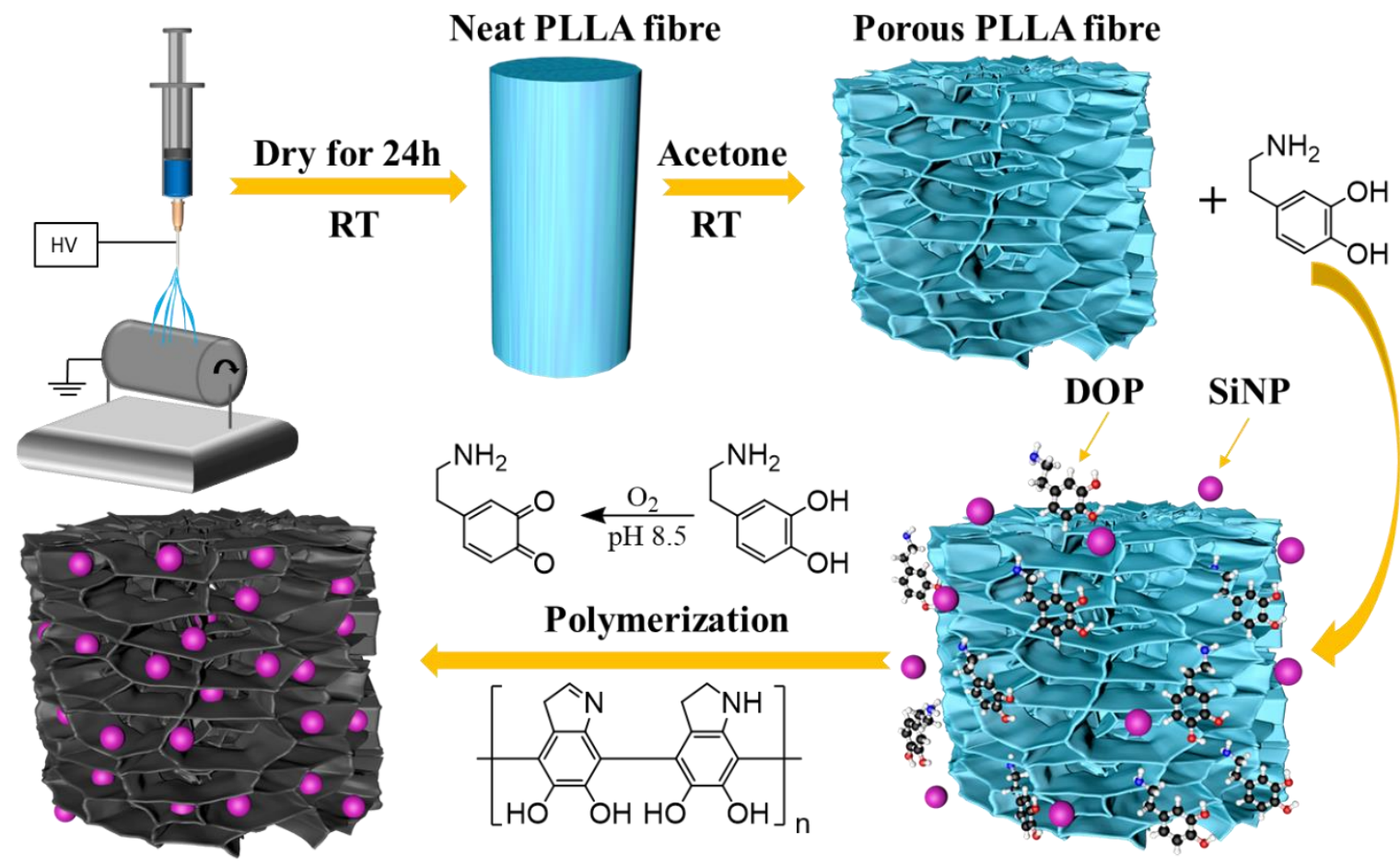

Figure 1. Illustration of porous structure formation of PLLA membrane by acetone treatment and DOP surface modification and SiNP coating on porous PLLA fibres.

\subsection{Synthesis of SiNPs}

SiNPs were synthesized based on our previous study [41]. In short, $100 \mathrm{~mL}$ EtOH, $8 \mathrm{~mL}$ ammonia and $3 \mathrm{ml}$ DI water were added into a $250 \mathrm{ml}$ glass flask which was heated in a water bath. When the temperature of the solution reached $60^{\circ} \mathrm{C}, 6 \mathrm{~mL}$ TEOS was added into the mixture with continuous stirring. The whole solution was kept at $60{ }^{\circ} \mathrm{C}$ for 3 hours. Finally, the nanoparticles were centrifuged and washed by DI water three times to remove any unreacted residuals. SiNPs were always kept under a wet condition to avoid agglomeration.

\subsection{Fabrication of PLLA/DOP/SiNP membranes}

PLLA/DOP/SiNP composite membranes were synthesized via a nanoparticle assistant strategy. A piece of porous PLLA membrane $\left(40 \times 80 \mathrm{~mm}^{2}\right)$ was immersed into $900 \mathrm{~mL}$ DOP 
solution $(2 \mathrm{mg} / \mathrm{mL})$ in a deep glass dish. The $\mathrm{pH}$ value of the solution was adjusted to 8.5 using Tris-buffer solution. Afterwards, the prepared SiNPs were added to the solution with required amounts and stirred under room temperature for 3 hours. In this work, three samples were prepared with different SiNPs concentrations, namely, $0.05 \mathrm{wt} \%, 0.10 \mathrm{wt} \%$ and $0.15 \mathrm{wt} \%$ of the whole reaction system. The linking between porous PLLA membrane, polydopamine (PDA) layer and SiNPs is mainly based on covalent and non-covalent bondings according to DOP oxidative selfpolymerization, as displayed in Figure 1. Finally, the resultant PLLA/DOP/SiNP porous membranes were rigorously washed using ethanol to remove un-linked SiNPs and any other impurities. These three samples were named porous PLLA/DOP/SiNP (0.05\%), porous PLLA/DOP/SiNP (0.10\%) and porous PLLA/DOP/SiNP (0.15\%), respectively. Porous PLLA/DOP fibrous membrane without SiNP was also prepared as control.

\subsection{Physicochemical structure characterizations}

The structural morphologies of membranes were observed using scanning electron microscopy (SEM, Ultra 55, Zeiss, Germany) at $1.5 \mathrm{kV}$ scanning voltage. All specimens were gold/palladium coated before imaging. The crystallization of specimens was analysed by X-ray diffraction spectroscopy (XRD, PANaytical X'Pert Pro). Fourier transform infrared spectroscopy (FTIR, NICOLET 5700, USA) was applied to confirm the chemical property of specimens. The elemental compositions of prepared membranes were examined by X-ray photoelectron spectroscopy (XPS, Kratos Axis Ultra, UK). The surface wettability of membranes was analysed by water-in-air contact angle (WCA) through drop shape analyser (DSA100, KRUSS, Germany).

\subsection{Mechanical property analysis}

The mechanical property of membranes was assessed using Instron 3344L3927 (USA) with a $10 \mathrm{~N}$ load cell. The tensile testing was performed at a constant speed of $5 \mathrm{~mm} / \mathrm{min}$. Samples were cut into a $5 \times 20 \mathrm{~mm}^{2}$ rectangular shape with $20 \mu \mathrm{m}$ thickness. Five pieces of each sample were tested and the analysis is based on the tensile stress-strain curves. 


\subsection{In vitro biological study}

Four circular membranes (diameter $=1.5 \mathrm{~cm}$ ) of each sample were cut for biological study. In vitro cytotoxicity and cell proliferation studies were conducted using human adipose-derived stem cells (hADSCs) (Invitrogen, USA). Cells were cultured in MesenPRO RS ${ }^{\mathrm{TM}}$ basal medium (Thermo Fisher Scientific, USA) in T75 cell culture flasks (Sigma-Aldrich, UK) under standard condition $\left(37^{\circ} \mathrm{C}, 5 \% \mathrm{CO}_{2}\right.$ concentration and $95 \%$ humidity) in a New Brunswick® Galaxy $170 \mathrm{R}$ incubator (Eppendorf, USA). Cells were harvested at approximate $80 \%$ confluency with $0.05 \%$ trypsin-EDTA (Invitrogen, USA) prior to cell seeding. Before cell seeding, all membranes were sterilized with $80 \%$ ethanol (Thermo Fisher Scientific, USA) for one hour, transferred to a 12-well plate (Cellstar®, Greiner Bio-One, Austria) and rinsed three times with Dulbecco's Phosphate Buffered Saline (PBS) (Thermo Fisher Scientific, USA). 20,000 cells (counted by Cellometer Auto 1000 Bright Field Cell Counter (Nexcelom Bioscience, USA)) in $1.0 \mathrm{~mL}$ corresponding medium were seeded on each sample and empty well as control group.

Cell viability and proliferation behaviour on membranes was evaluated at 1,3 and 7 days after cell seeding using Alamar Blue assay. At each particular time point, $1.0 \mathrm{~mL}$ of medium containing 0.001\% Alamar Blue (Sigma-Aldrich, UK) was added to each well and incubated for 4 hours. 150 $\mu \mathrm{L}$ liquid sample from each well was then transferred into a 96-well plate and the fluorescence intensity was measured by a multi-detection microplate reader Synergy HT (BioTec, USA) (excitation wavelength of $540 \mathrm{~nm}$ and emission wavelength of $590 \mathrm{~nm}$ ).

Cell attachment status and morphology were further assessed using laser confocal microscopy, with cell membranes and nuclei stained. After day 7, cell seeded samples were fixed using $10 \%$ neutral buffered formalin (Sigma-Aldrich, UK) for 60 minutes. 0.1\% Triton X-100 (Sigma-Aldrich, UK) was used to permeabilize cell membrane, washed twice with PBS, and then immersed for 30 minutes in an immunocytochemistry blocking buffer comprised 5\% fetal bovine serum in PBS. Samples were again rinsed twice in PBS. Finally, samples were stained with 4',6-Diamidine-2'- 
phenylindole dihydrochloride (Invitrogen ${ }^{\mathrm{TM}}$, ThermoFisher Scientific, MA, USA) and Alexa Fluor

TM 488 Phalloidin (Invitrogen ${ }^{\mathrm{TM}}$, ThermoFisher Scientific, MA, USA) at the manufacturer recommended concentration and incubation time. Images were obtained on a Leica TCS SP8 (Leica, Milton Keynes, UK) laser confocal microscopy.

\subsection{Statistical analysis}

Analysis of XRD results, chemical property, mechanical properties and wettability were performed by Origin 2018 software (OriginLab corporation, USA). Significance was calculated by Prism 8 software (GraphPad software, USA) using a one-way ANOVA significance measurement combined with Tukey multiple comparisons testing.

\section{Results and discussions}

\subsection{Porous PLLA fibrous membranes}

For tissue engineering scaffolds, their surface plays a very important role for cell attachment and proliferation. Scaffolds with high surface area can provide more attachment sites for cells and contact areas with surrounding tissues which all benefit tissue repairment and regeneration. Having high surface area is an inherent advantage for electrospun nano/micro polymer fibres and fibrous membranes. This advantage has enabled and promoted very extensive researches and applications of electrospun scaffolds in a wide range of tissue engineering fields. A lot of researchers have developed various methods and mechanisms to further improve the surface area of electrospun fibres, such as breath figures, phase separation, removal of specific polymer or particles and enhancing crystallization through introduced solvent [42-46]. Through these efforts, the prepared electrospun products could gain more specific surface area. However, further research is needed to achieve even higher surface area. Additionally, compared with smooth surface, nano/micro fibres with rough surface are more suitable for further surface modification. The irregular surface can provide more anchor points for coating materials and improve coating incorporation. 
PLLA fibres, like most of other electrospun polymer fibres, present a solid and smooth surface, as displayed in Figure 2(a). Numerous studies have demonstrated that this kind of fibrous structure is a good substrate for cell seeding and culturing. However, they are not an ideal substrate for nano particles, especially $\mathrm{SiO}_{2}$ nano spheres in this study. The smooth convex surface of a solid fibre provides only a very small contact point for each nano sphere which limits and weakens their adhesion strength. Without strong adhesion, nano spheres will be easily peeled off from substrates during the subsequent biomedical processes. Therefore, it is necessary to change the smooth fibre surface to rougher or porous surface for higher coating strength of nano spheres.

In order to increase the surface area of PLLA fibres, a post-treatment process has been developed to fabricate porous structures by our group [20, 21]. After PLLA fibres were electrospun and collected, they were immersed into acetone for 5 minutes at room temperature. While acetone induced the recrystallization of PLLA phase, countless small holes were generated throughout the whole fibres, as shown in Figure 2(b). The porous fibres had about twice the diameter of the original ones (Figure 2(c)). The pore size ranged from $300 \mathrm{~nm}$ to $500 \mathrm{~nm}$.

A dual-solvent system was selected to prepare the electrospinning solution. DCM and DMF acted as the solvent and non-solvent of PLLA, respectively. And more importantly, this solvent system was specially designed to control PLLA phases and crystalline structures. Before electrospinning, PLLA chains were random coils in the solution. While the stream of solution flied from the needle tip to the collector, it was stretched and elongated. At the same time, the solvents were evaporated rapidly. With faster evaporating rate, almost all of DCM was removed when the fibres reached the collector, but some of DMF was still trapped inside. As a non-solvent of high molecular weight PLLA, DMF was separated from PLLA phase and stayed within the PLLA matrix. After the fibres were completely dried, the space occupied by DMF left voids and phase interfaces inside the PLLA fibres. When these fibres were immersed in acetone, these voids and phase interfaces guided acetone to fast permeate into the PLLA matrix. While acetone entered and swelled amorphous PLLA phases, it induced the rearrangement of PLLA chains at room temperature. As a 
result of recrystallization of PLLA phases, a highly porous structure was generated throughout the whole PLLA fibre, as shown in Figure 1. It is expected that the porous fibres with higher specific surface area are more suitable for coating materials than solid fibres.

During electrospinning, the fast evaporation of solvent left insufficient time for PLLA chains to reach a high crystallinity. Most of the polymer was in an amorphous state which was verified by XRD curves (Figure 2(d)). No obvious crystallization peaks could be observed for electrospun PLLA fibres. However, after PLLA fibres were treated by acetone, four obvious crystallization peaks were observed which are mainly formed from (010), (110), (200), (203) and (015) planes, respectively $[47,48]$. Particularly, the peak at $16.8^{\circ}$ shows the highest intensity. These results indicate that acetone treatment leeds to the recrystallization of PLLA fibres.

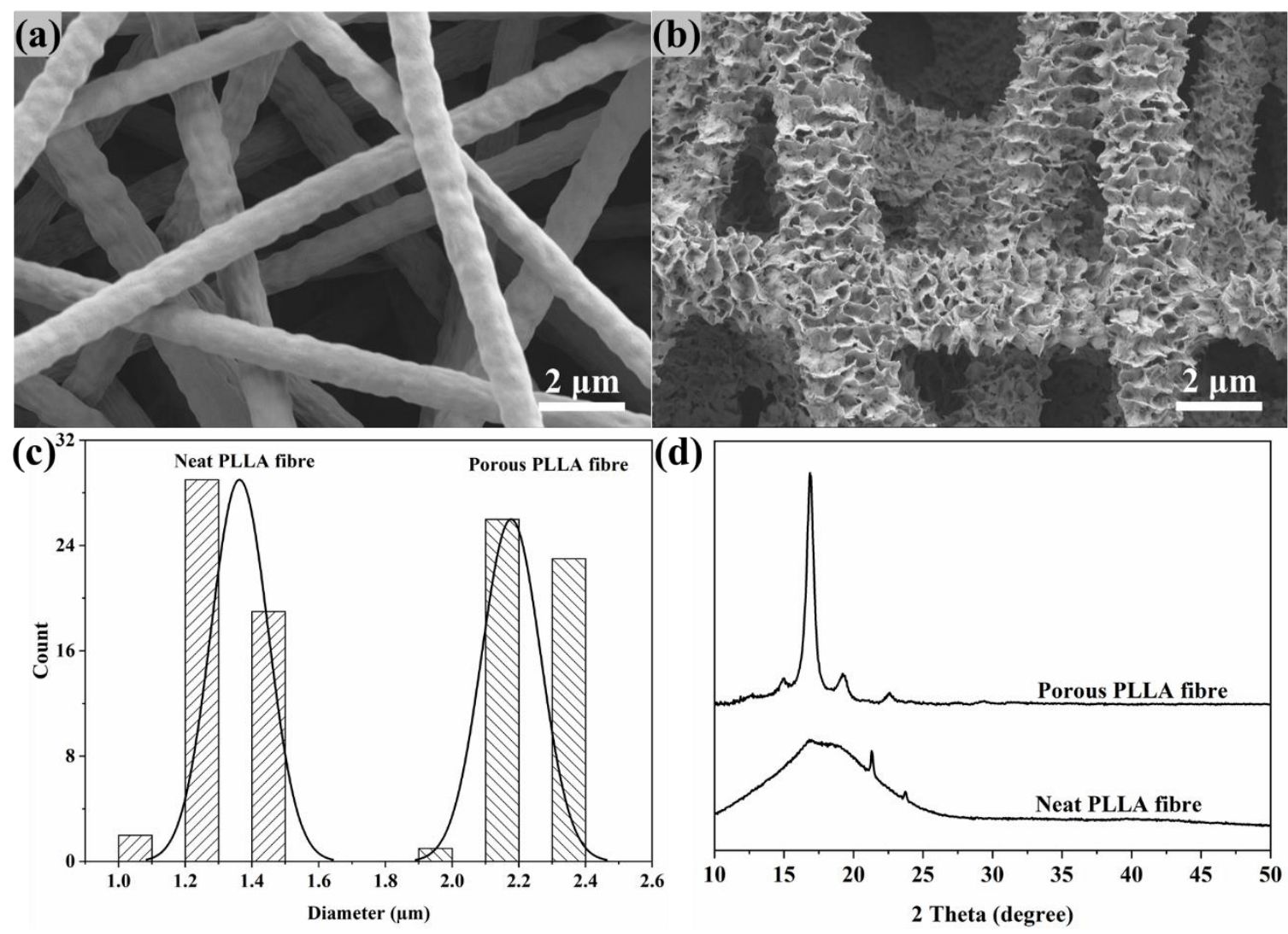

Figure 2. SEM of neat PLLA fibres (a) and porous PLLA fibres (b). Diameter distribution of neat and porous PLLA fibres (c). XRD spectrum of neat and porous PLLA fibres (d). 


\subsection{SiNP coating}

The normal straightforward strategy to introduce functional nanoparticles into electrospun fibres is to directly mix them with polymer solution. However, particle aggregation always happens and decreases the dispersion of particles inside polymer fibres. Moreover, only a very small number of particles can function when they are coincidentally exposed on the fibre surface. Most of the particles are completely covered by polymers and cannot react with the external environment. To address these issues, SiNPs was coated on fibre surface for better efficiency in this study.

Based on the pore size on porous PLLA fibres (about $300-500 \mathrm{~nm}$ ), the diameter of SiNPs was controlled at about $150 \mathrm{~nm}$ to ensure they can be effectively coated into the pores on PLLA fibres, as shown in Figure 3(a). Too small particles may affect their bio-properties and influence the final cell attachment. On the other hand, too large particles cannot be loaded into pores and affect the interaction between fibres and particles.

There is little apparent physical or chemical connection between PLLA fibres and SiNPs if they simply contact with each other. Therefore, DOP was used to link them together for stronger coating interface. Surface modification of porous PLLA/DOP/SiNP composite fibres was obtained by dissolving DOP and dispersing SiNPs in DI water at $\mathrm{pH} 8.5$ with vigorous stirring. The prepared solution was turned from transparent to light pink and finally became dark brown. This reaction is attributed to the dopamine oxidative self-polymerization.

This approach, developed by Lee et al., could produce the PDA adhesion functional layer on various organic and inorganic substrates $[34,49,50]$. In this work, PDA adhesion layer with functional groups was firstly produced on the porous PLLA fibres and then the SiNPs were coated onto the PLLA fibres through these functional groups. Therefore, the chemical binding between particles and fibres could significantly enhance their incorporation. It is worth emphasizing that DOP coating had nearly no effect on the porous structure (Figure 3(b)).

For dry nano particles, it is always a challenge to disperse them in liquid. To avoid the aggregation of SiNPs, they were always kept in a wet state from the initial synthetic process to the 
final coating process. For PLLA fibres with less SiNPs, a lot of isolated spheres can be observed on the fibre surface (Figure 3(c)). Even for PLLA fibres with more SiNPs, countless particles are closed packed on the fibre suerface, but no aggregation is seen, as shown in Figure $3(\mathrm{~d} \&$ e). As expected, more SiNPs are coated onto the fibres with the increasing of their concentration in the coating solution. It is worth stressing that all samples were rigorously washed using ethanol after the coating process. Although it is difficult to quantitatively measure the coating strength, we believe that SiNP coating is strong enough for any further biomedical process. The uniform distribution of SiNPs may significantly improve cell attachment and proliferation.

Four obvious peaks from XRD curve further indicate that DOP surface modification does not affect the crystallization of PLLA membranes (Figure 3(f)). Additionally, the four obvious peaks remain with increasing of SiNP loading. Moreover, the peak at $22.5^{\circ}$, indicating SiNP, becomes more obvious for porous PLLA/DOP/SiNP $(0.10 \%)$ and porous PLLA/DOP/SiNP $(0.15 \%)$. The peak intensity increases with the increasing of SiNP concentration. These results demonstrate that porous PLLA membrane modified through DOP and SiNP coating can maintain high surface roughness, high surface area and high crystallization, which offer high potential for periosteum application in the field of bone tissue engineering. 

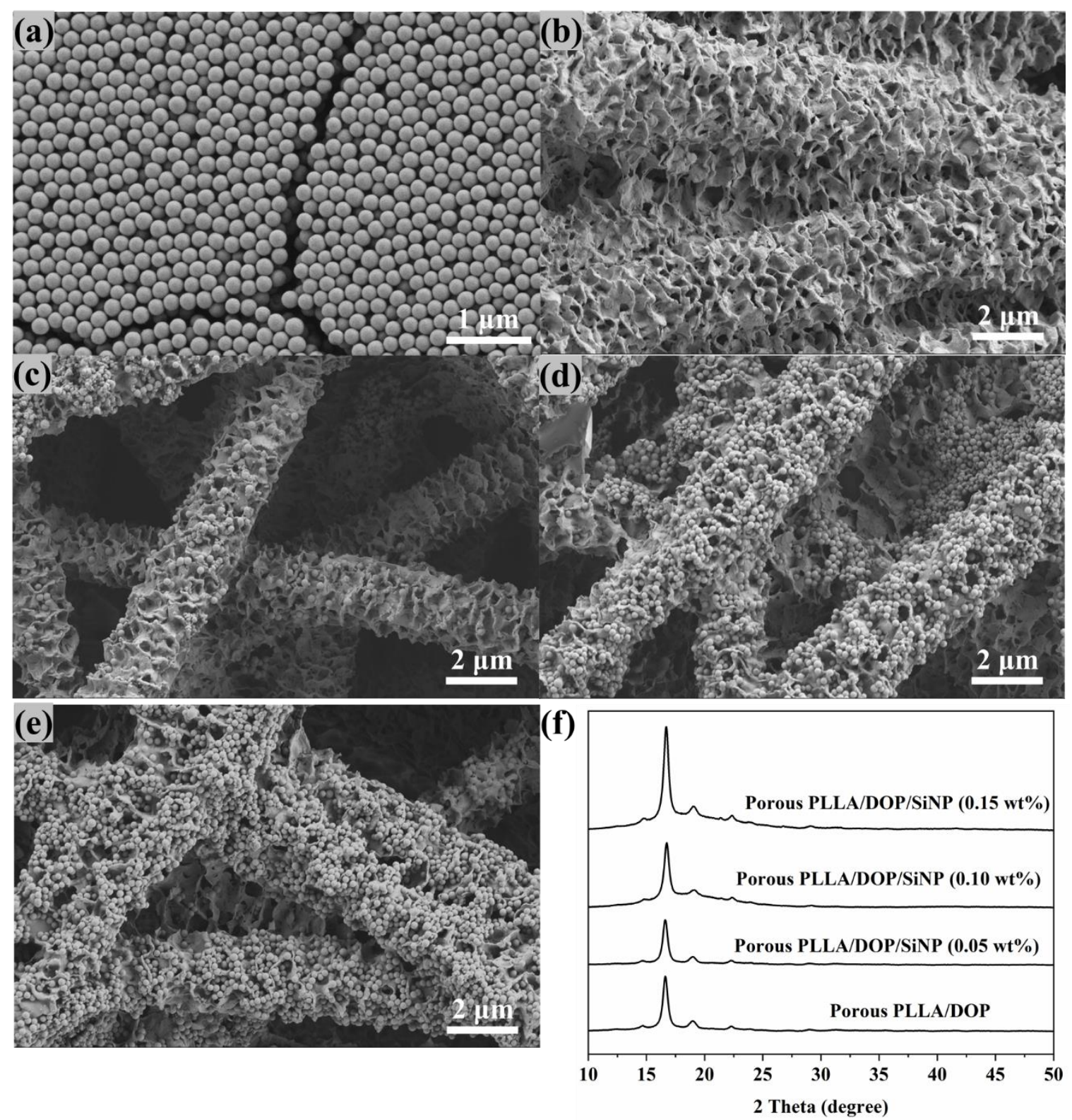

Figure 3. SEM images of produced SiNPs (a), porous PLLA membrane after DOP surface modification (b), and porous PLLA/DOP/SiNP membranes with different SiNP concentrations $(0.05,0.10$ and $0.15 \mathrm{wt} \%)$ (c-e); XRD curves of porous PLLA/DOP and PLLA/DOP/SiNP membranes (f).

\subsection{Chemical property evaluation of membranes}

FTIR spectra show obvious peaks relevant to functional groups of PLLA, DOP and SiNP (Figure 4(a)), which further indicate the successful preparation of porous PLLA/DOP/SiNP 
membranes. The porous PLLA membrane spectrum shows that the bands at about $1750 \mathrm{~cm}^{-1}$ and $1400 \mathrm{~cm}^{-1}$ are relative to $-\mathrm{C}=\mathrm{O}$ group stretching and $\mathrm{CH}_{3}$ group bending vibrations, respectively. Asymmetrical, symmetrical and stretching C-O-C group vibration appear between $1000 \mathrm{~cm}^{-1}$ and $1300 \mathrm{~cm}^{-1}$, respectively [51]. The distinct peaks between $950 \mathrm{~cm}^{-1}$ and $1100 \mathrm{~cm}^{-1}$ are identified as Si-O-Si group [52], which confirms that SiNPs are successfully incorporated into the porous PLLA membranes. With increasing SiNP loading, the peak of Si-O-Si group becomes more obvious and the intensity of special peaks of PLLA membrane is reduced. The chemical property of composite membrane is further investigated by XPS (Figure 4(b)). For the porous PLLA membrane, only O 1s and $\mathrm{C}$ 1s peaks are detected. For the XPS pattern of porous PLLA/DOP and PLLA/DOP/SiNP, they both have a typical $\mathrm{N} 1 \mathrm{~s}$ peak at around $400 \mathrm{eV}$ which is mainly related to the DOP. Furthermore, for the porous PLLA/DOP/SiNP membrane, Si 2s and Si 2p peaks are also detected which belong to SiNP. These XPS patterns suggest that SiNPs are successfully coated onto the porous PLLA membranes through the DOP layer.
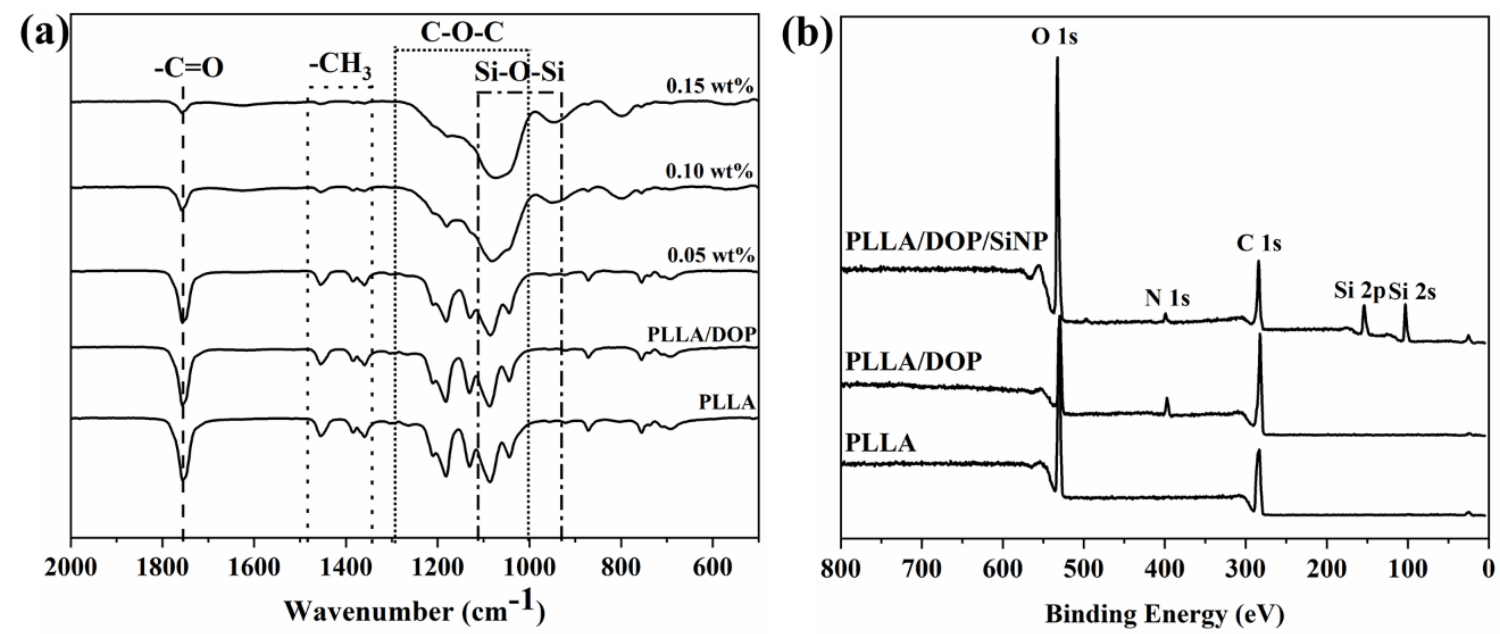

Figure 4. Chemical structure analysis of membranes. (a) FTIR spectrum of prepared samples; (b) XPS pattern of porous PLLA membrane, porous PLLA/DOP membrane and porous PLLA/DOP/SiNP membrane (0.15 wt $\%)$. 


\subsection{Mechanical property of membranes}

Mechanical properties of bio-membranes are significant factors to demonstrate their potential in biomaterial applications. The mechanical properties of prepared composite membranes are examined by the measurement of tensile properties, as displayed in Figure 5(a). For the neat PLLA membrane, the Young's modulus is the lowest $(14.77 \pm 1.25 \mathrm{MPa})$, however, the strain can reach $63.47 \pm 1.27 \%$. The acetone treatment can enhance Young's modulus of PLLA membrane to 5 times that of neat PLLA membrane (red curve). After DOP grafting, the coated porous PLLA membrane shows lower stress $(3.01 \pm 0.32 \mathrm{MPa})$ and strain $(41.39 \pm 1.75 \%)$. With the increase of SiNP loading, the breaking stress $(5.54 \pm 0.35 \mathrm{MPa})$ and strain $(64.17 \pm 0.23 \%)$ of PLLA/DOP/SiNP are improved, as displayed in porous PLLA/DOP/SiNPs (0.05 wt\%) curve. Results also suggest more SiNP coating on PLLA fibres reduces their breaking stress to 4.79 $\mathrm{MPa}$ and 3.75 MPa respectively. Their corresponding strains are also reduced. Therefore, DOP grafting and SiNP loading effectively enhance the strength of PLLA/DOP/SiNP.

\subsection{Wettability of membranes}

Wettability is significant for materials used in the field of bone regeneration it influences the cell affinity. As usual, hydrophilic materials show higher cell attachment than hydrophobic materials. The water-in-air contact angle (WCA) was measured to evaluate the effect of DOP grafting and SiNP loading to the wettability of composite membranes (Figure 5(b)). Results reveal that the neat PLLA membrane and porous PLLA membrane have hydrophobic surface with 136.97 $\pm 1.50^{\circ}$ and $133.90 \pm 1.25^{\circ}$ contact angle, respectively. After DOP grafting, the WCA of treated porous PLLA/DOP membrane is dramatically decreased to $118.70 \pm 1.84^{\circ}$, which is ascribed to the hydrophilic DOP. Furthermore, as for the porous PLLA/DOP/SiNPs membranes, the value of WCA decreases continually to $110.87 \pm 1.34^{\circ}, 99.10 \pm 0.40^{\circ}$ and $96.37 \pm 1.22^{\circ}$ with the increasing of SiNPs. Therefore, DOP grafting and SiNP loading effectively improve the wettability of PLLA/DOP/SiNP, which is beneficial for cell seeding. 

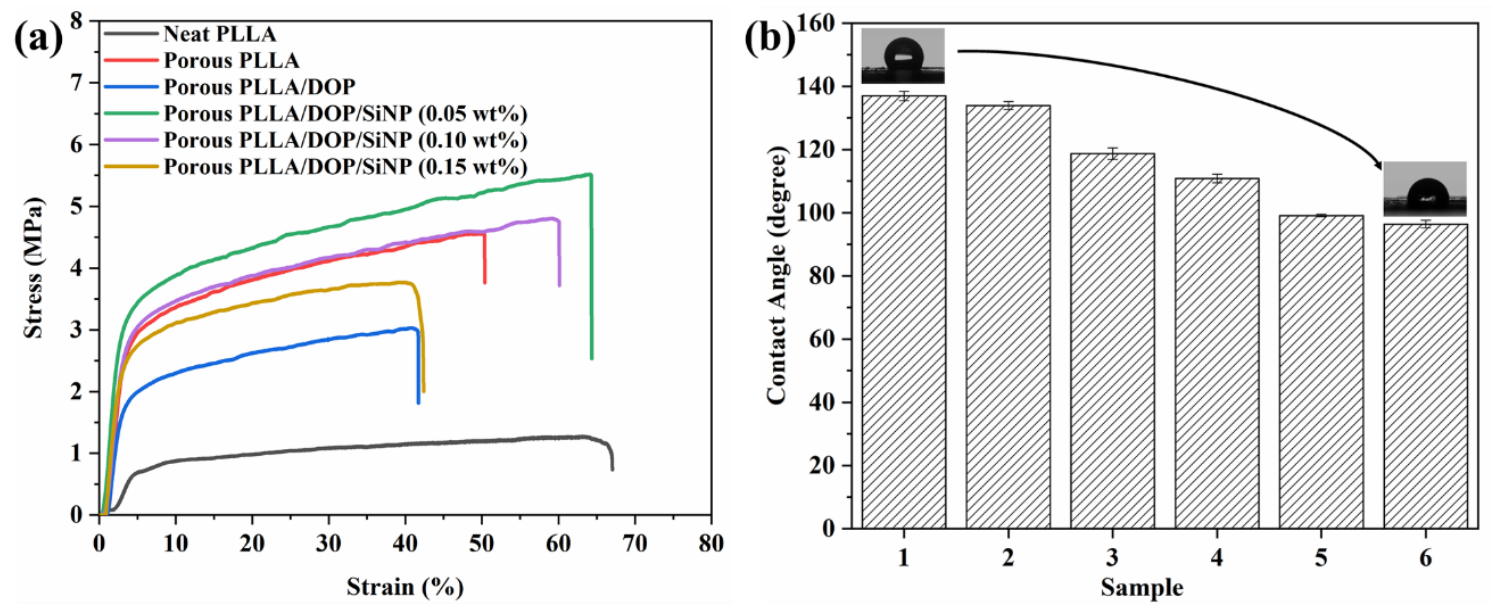

Figure 5. Mechanical property (a) and wettability analysis (b) of PLLA membranes. In the WCA chart, 1: neat PLLA, 2: porous PLLA, 3: porous PLLA/DOP, 4: porous PLLA/DOP/SiNP (0.05 wt\%), 5: porous PLLA/DOP/SiNP (0.10 wt\%) and 6: porous PLLA/DOP/SiNP $(0.15 \mathrm{wt} \%)$.

\subsection{Cell viability and proliferation}

Figure 6 shows membrane samples before and after cell culture. It is obvious that the original colour of porous PLLA membranes is white, and they change to dark brown after DOP surface modification (Figure $(\mathrm{a} \& \mathrm{~b})$ ). Both SEM and confocal microscopy images show that the membranes can support cell attachment and proliferation. After day 7, cells were fully embedded within porous fibres (Figure 6 (c \& d)). Extensive cell attachment and spreading can be observed (Figure 6 (e \& f)). Images also show that porous PLLA/DOP/SiNP (0.05 wt $\%)$ composite membrane has slightly better cell confluence than porous PLLA membrane.

Cytotoxicity/cell proliferation evaluation results are presented in Figure 7, with fluorescence intensity values measured at different time points for different membranes. The fluorescence intensity is proportional to the number of metabolically active cells. For porous PLLA membrane, its fluorescence intensity values decrease from day 1 to day 7 after cell seeding, which suggests that the membrane supports cell attachment but the surface morphology does not promote cell proliferation. Contrary to this result, after DOP grafting, porous PLLA/DOP membranes show 
higher fluorescence intensity than PLLA membranes, which indicates that porous PLLA membrane with DOP surface modification also shoes better cellular biocompatibility.

For PLLA/DOP/SiNP membranes $(0.05,0.10$ and $0.15 \mathrm{wt} \%)$, significantly higher cell attachment and proliferation can also be observed from day 1 to day 7, comparing with porous PLLA membrane. The highest cell proliferation is observed at day 3 with $0.10 \mathrm{wt} \%$ PLLA/DOP/SiNP membrane. The fluorescence intensity values drop on day 7 compared with previous two time points, this due to lower metabolic activity caused by cell over confluency. This fact is also proved by confocal microscopy images in Figure 6.

These results show that the addition of dopamine and SiNP loading have certain improvement effect on the attachment and proliferation of hADSCs, while the combination of dopamine grafting and $0.10 \mathrm{wt} \%$ SiNP shows the most significant improvement. The improved cell viability and proliferation may be attributed to dopamine grafting and the enhanced surface stiffness of porous PLLA/DOP/SiNP composite membranes [53]. Moreover, the enhancement in hydrophilicity caused by SiNP loading may also benefit the cell attachment and viability during cell seeding and early cell proliferation/spreading stage, resulted improved biological performance [52].

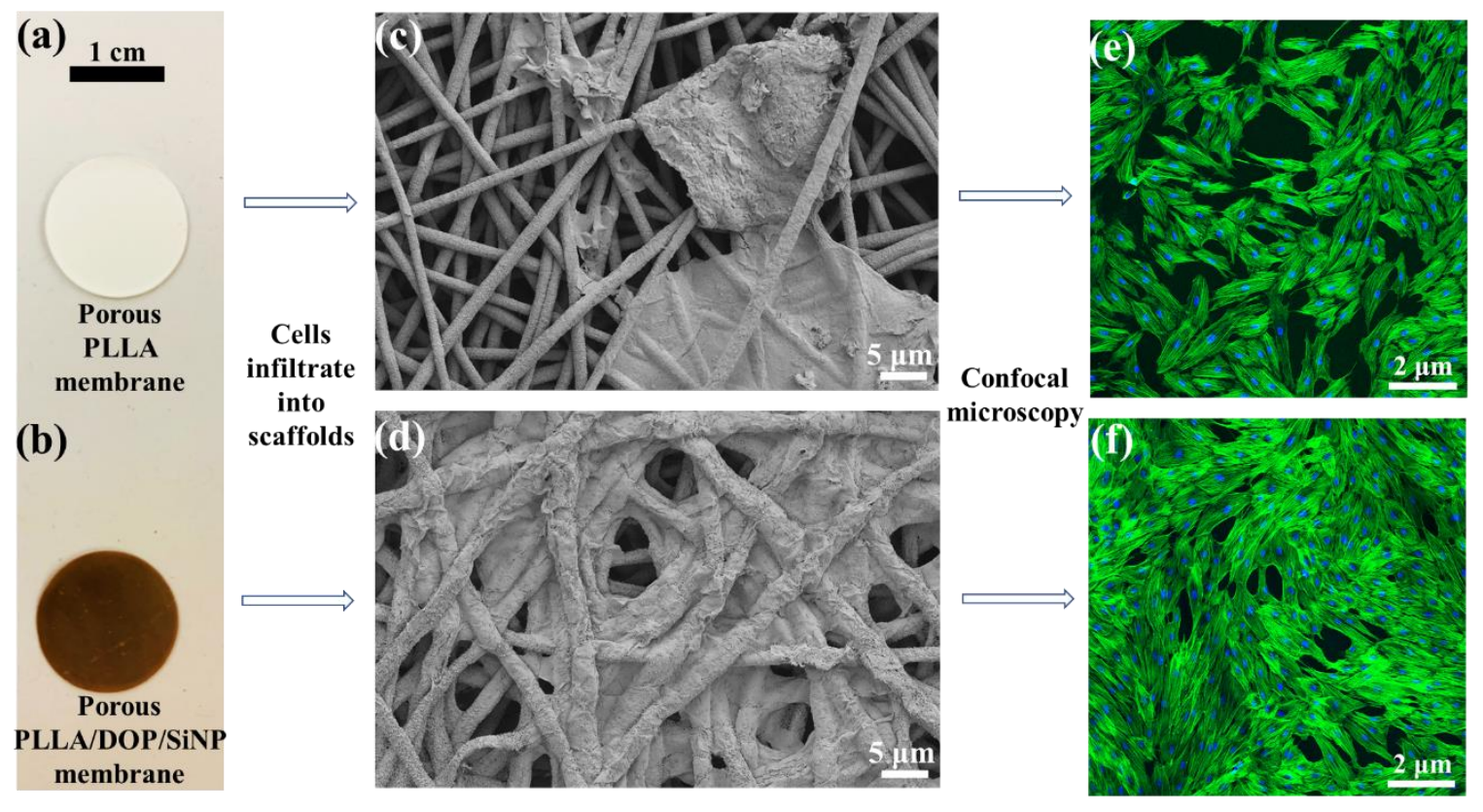


Figure 6. Membranes as-fabricated, porous PLLA membrane (a) and porous PLLA/DOP/SiNP composite membrane (b); SEM images (c \& d) and confocal microscopy images (e and f) of porous PLLA membrane and porous PLLA/DOP/SiNP (0.05 wt $\%$ ) composite membrane after 7 days cell culture.

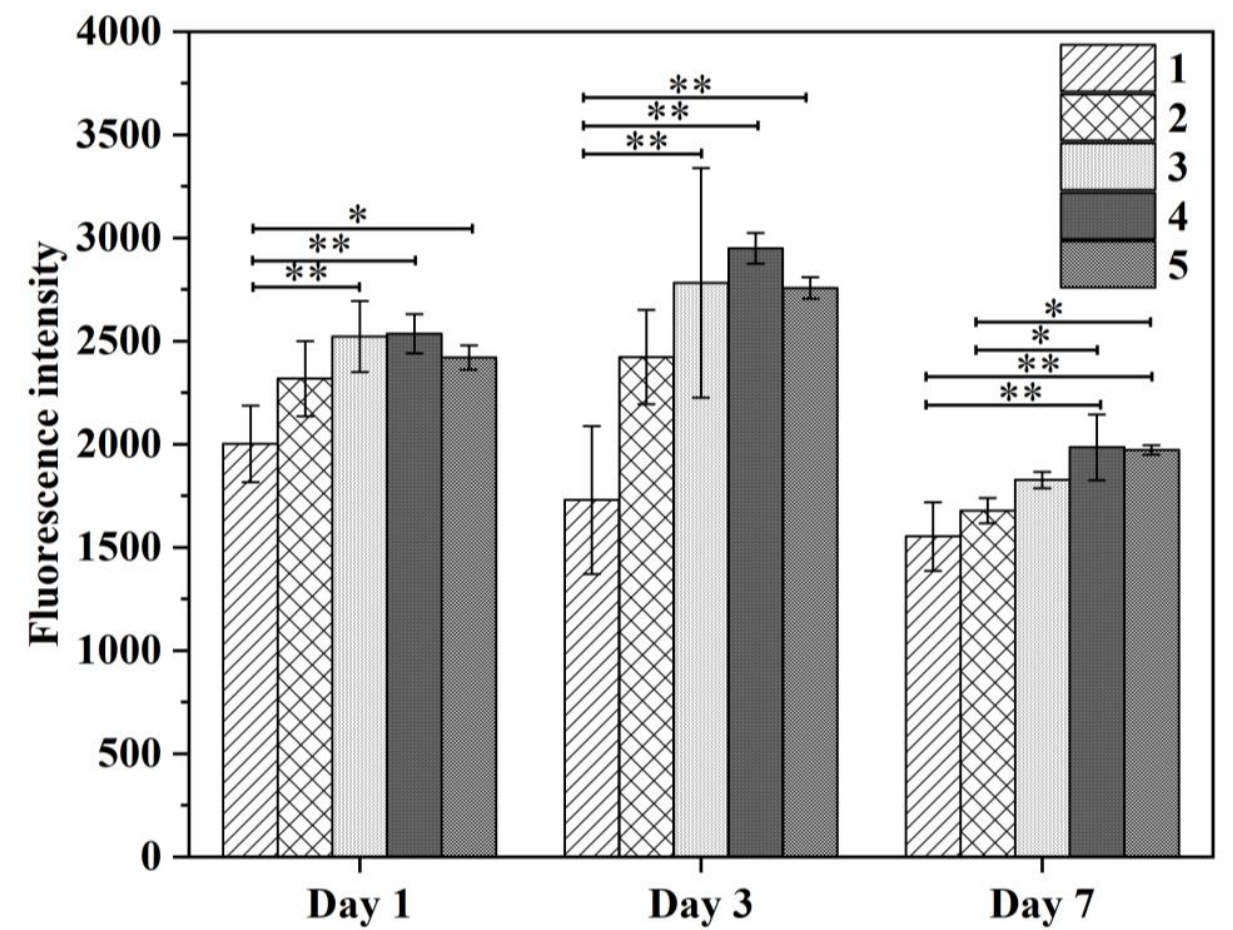

Figure 7. Cell viability/proliferation results (fluorescence intensity) of membranes after 1, 3 and 7 days of cell culture $(* \mathrm{p}<0.05, * * \mathrm{p}<0.01)$. 1-neat PLLA, 2-porous PLLA, 3-porous PLLA/DOP, 4porous PLLA/DOP/SiNP (0.05 wt\%), 5-porous PLLA/DOP/SiNP (0.10 wt\%) and 6- porous PLLA/DOP/SiNP $(0.15 \mathrm{wt} \%)$.

\section{Conclusions}

This work mainly focuses on the validation of the concept of using membrane structure with the integration of biomolecules and stem cell to solve the problem of periosteum regeneration, and focusing on the research on material combination as well. Highly porous PLLA fibres, with higher surface area and rougher surface, are fabricated by electrospinning and acetone treatment, and used 
as substrates for nano particle coating. With the help of DOP, SiNPs are strongly and uniformly coated on the fibre surface, which increases the hydrophilicity of PLLA/DOP/SiNP composite membranes. The membrane mechanical properties are also improved. As expected, PLLA/DOP/SiNP membrane shows good biocompatibility and improved cell attachment and proliferation. This novel porous structure and the multifunctional properties make the composite membrane a promising candidate for periosteum in the field of bone regeneration.

\section{Acknowledgement}

Weiguang Wang wishes to acknowledge the funding Rosetrees \& Stoneygate Trust Young Enterprise Fellowship (Ref: A2750/M874) provided by Rosetrees Trust (charity number 298582) and Stoneygate Trust (charity number 1119976). This research has also been supported by The University of Manchester and the Engineering and Physical Sciences Research Council (EPSRC) of the UK, the Global Challenges Research Fund (GCRF), grant number EP/R01513/1. Jing Zhang acknowledges Science and Technology Project of Shaanxi, China (Grant No: 2017JQ2006), and

the youth talent promotion plan of Shaanxi Association for Science and Technology (Grant No:20190305). We acknowledge the support of the Electron Microscopy Centre and XRD suite in The University of Manchester.

\section{References}

[1] W. Wang, J.R.P. Junior, P.R.L. Nalesso, D. Musson, J. Cornish, F. Mendonça, G.F. Caetano, P. Bártolo, Engineered 3D printed poly( $\varepsilon$-caprolactone)/graphene scaffolds for bone tissue engineering, Materials Science \& Engineering C 100 (2019) $759-770$.

[2] M.M. Stevens, Biomaterials for bone tissue engineering, Materials Today 11(5) (2008) 18-25. 
[3] E. Xie, Y. Hu, X. Chen, X. Bai, D. Li, L. Ren, Z. Zhang, In vivo bone regeneration using a novel porous bioactive composite, Applied Surface Science 255(2) (2008) 545-547.

[4] C.-A. Dascălu, A. Maidaniuc, A.M. Pandele, S.I. Voicu, T. Machedon-Pisu, G.E. Stan, A. Cîmpean, V. Mitran, I.V. Antoniac, F. Miculescu, Synthesis and characterization of biocompatible polymer-ceramic film structures as favorable interface in guided bone regeneration, Applied Surface Science 494 (2019) 335-352. [5] G. Ellender, S.A. Feik, B.J. Carach, Periosteal structure and development in a rat caudal vertebra, Journal of anatomy 158 (1988) 173-187.

[6] T. Wang, Y. Zhai, M. Nuzzo, X. Yang, Y. Yang, X. Zhang, Layer-by-layer nanofiber-enabled engineering of biomimetic periosteum for bone repair and reconstruction, Biomaterials 182 (2018) 279-288.

[7] Q. Wang, J. Xu, H. Jin, W. Zheng, X. Zhang, Y. Huang, Z. Qian, Artificial periosteum in bone defect repair-A review, Chinese Chemical Letters 28(9) (2017) 1801-1807.

[8] W. Liu, W. Bi, Y. Sun, L. Wang, X. Yu, R. Cheng, Y. Yu, W. Cui, Biomimetic organic-inorganic hybrid hydrogel electrospinning periosteum for accelerating bone regeneration, Materials Science \& Engineering C 110 (2020).

[9] A.K. Jaiswal, S.S. Kadam, V.P. Soni, J.R. Bellare, Improved functionalization of electrospun PLLA/gelatin scaffold by alternate soaking method for bone tissue engineering, Applied Surface Science 268 (2013) 477-488.

[10] L. Roseti, V. Parisi, M. Petretta, C. Cavallo, G. Desando, I. Bartolotti, B. Grigolo, Scaffolds for Bone Tissue Engineering: State of the art and new perspectives, Materials Science \& Engineering C 78 (2017) 1246-1262. 
[11] M. Gong, C. Chi, J. Ye, M. Liao, W. Xie, C. Wu, R. Shi, L. Zhang, Icariinloaded electrospun PCL/gelatin nanofiber membrane as potential artificial periosteum, Colloids and Surfaces B: Biointerfaces 170 (2018) 201-209.

[12] N. Udomluck, H. Lee, S. Hong, S.-H. Lee, H. Park, Surface functionalization of dual growth factor on hydroxyapatite-coated nanofibers for bone tissue engineering, Applied Surface Science 520 (2020).

[13] D. Lee, D.N. Heo, S.J. Lee, M. Heo, J. Kim, S. Choi, H.-K. Park, Y.G. Park, H.N. Lim, I.K. Kwon, Poly(lactide-co-glycolide) nanofibrous scaffolds chemically coated with gold-nanoparticles as osteoinductive agents for osteogenesis, Applied Surface Science 432(PB) (2018) 300-307.

[14] A. Rahmani Del Bakhshayesh, E. Mostafavi, E. Alizadeh, N. Asadi, A. Akbarzadeh, S. Davaran, Fabrication of Three-Dimensional Scaffolds Based on Nano-biomimetic Collagen Hybrid Constructs for Skin Tissue Engineering, ACS omega 3(8) (2018) 8605-8611.

[15] A. Castro, M. Diba, M. Kersten, J. Jansen, J. van Den Beucken, F. Yang, Development of a PCL-silica nanoparticles composite membrane for Guided Bone Regeneration, Materials Science \& Engineering. C, Biomimetic Materials, Sensors and Systems 85 (2018) 154.

[16] Y.-F. Goh, M. Akram, A. Alshemary, R. Hussain, Antibacterial polylactic acid/chitosan nanofibers decorated with bioactive glass, Applied Surface Science 387 (2016) $1-7$.

[17] Q.P. Pham, U. Sharma, A.G. Mikos, Electrospinning of polymeric nanofibers for tissue engineering applications: a review, Tissue engineering 12 (5) (2006) 1197 1211.

[18] D. Depan, B. Girase, J.S. Shah, R.D.K. Misra, Structure-process-property relationship of the polar graphene oxide-mediated cellular response and stimulated 
growth of osteoblasts on hybrid chitosan network structure nanocomposite scaffolds, Acta Biomaterialia 7(9) (2011) 3432-3445.

[19] A. Cheng, Z. Schwartz, A. Kahn, X. Li, Z. Shao, M. Sun, Y. Ao, B. Boyan, H. Chen, Advances in Porous Scaffold Design for Bone and Cartilage Tissue Engineering and Regeneration, Tissue Engineering Part B: Reviews 25(1) (2019) 14 29.

[20] J. Song, B. Zhang, Z. Lu, Z. Xin, T. Liu, W. Wei, Q. Zia, K. Pan, R.H. Gong, L. Bian, Y. Li, J. Li*, Hierarchical Porous Poly(l-lactic acid) Nanofibrous Membrane for Ultrafine Particulate Aerosol Filtration, ACS Applied Materials \& Interfaces 11(49) (2019) 46261-46268.

[21] Q. Zia, M. Tabassum, Z. Lu, M.T. Khawar, J. Song, H. Gong, J. Meng, Z. Li, J. Li, Porous poly(L-lactic acid)/chitosan nanofibres for copper ion adsorption, Carbohydrate Polymers 227(1) (2019).

[22] W.-B. Tsai, W.-T. Chen, H.-W. Chien, W.-H. Kuo, M.-J. Wang, Poly(dopamine) coating of scaffolds for articular cartilage tissue engineering, Acta Biomaterialia 7(12) (2011) 4187-4194.

[23] H. Kang, K. Zhang, H.J. Jung, B. Yang, X. Chen, Q. Pan, R. Li, X. Xu, G. Li, V.P. Dravid, L. Bian, An In Situ Reversible Heterodimeric Nanoswitch Controlled by Metal-Ion-Ligand Coordination Regulates the Mechanosensing and Differentiation of Stem Cells, Advanced Materials 30(44) (2018) n/a-n/a.

[24] H. Kang, B. Yang, K. Zhang, Q. Pan, W. Yuan, G. Li, L. Bian, Immunoregulation of macrophages by dynamic ligand presentation via ligand-cation coordination, Nature communications 10(1) (2019) 1696-1696.

[25] R. Li, S. Lin, M. Zhu, Y. Deng, X. Chen, K. Wei, J. Xu, G. Li, L. Bian, Synthetic presentation of noncanonical Wnt5a motif promotes mechanosensing-dependent differentiation of stem cells and regeneration, Science Advances 5(10) (2019). 
[26] X. Zhou, W. Weng, B. Chen, W. Feng, W. Wang, W. Nie, L. Chen, X. Mo, J. $\mathrm{Su}, \mathrm{C}$. He, Mesoporous silica nanoparticles/gelatin porous composite scaffolds with localized and sustained release of vancomycin for treatment of infected bone defects, Journal of Materials Chemistry B 6(5) (2018) 740-752.

[27] J.L. Paris, N. Lafuente-Gómez, M.V. Cabañas, J. Román, J. Peña, M. ValletRegí, Fabrication of a nanoparticle-containing 3D porous bone scaffold with proangiogenic and antibacterial properties, Acta Biomaterialia 86 (2019) 441 -449.

[28] M.T. Tavares, M.B. Oliveira, J.F. Mano, J.P.S. Farinha, C. Baleizão, Bioactive silica nanoparticles with calcium and phosphate for single dose osteogenic differentiation, Materials Science \& Engineering C 107 (2020).

[29] R. Eivazzadeh-Keihan, K.K. Chenab, R. Taheri-Ledari, J. Mosafer, S.M. Hashemi, A. Mokhtarzadeh, A. Maleki, M.R. Hamblin, Recent advances in the application of mesoporous silica-based nanomaterials for bone tissue engineering, Materials Science \& Engineering C 107 (2020).

[30] M. Karimi, H. Mirshekari, M. Aliakbari, P. Sahandi-Zangabad, M. Hamblin, Smart mesoporous silica nanoparticles for controlled-release drug delivery, Nanotechnology Reviews 5(2) (2016) 195-207.

[31] K. Chan, J.K.-H. Tsoi, O.-K. Wu, M.J.-Y. Yon, R.W.-K. Wong, Mechanical and biological evaluations of novel electrospun PLLA composite scaffolds doped with oxide ceramics, Journal of the Mechanical Behavior of Biomedical Materials 97 (2019) 229-237.

[32] N. Ganesh, R. Jayakumar, M. Koyakutty, U. Mony, S.V. Nair, Embedded silica nanoparticles in poly(caprolactone) nanofibrous scaffolds enhanced osteogenic potential for bone tissue engineering, Tissue engineering. Part A 18(17-18) (2012) $1867-1881$. 
[33] M. Mehrasa, M.A. Asadollahi, B. Nasri-Nasrabadi, K. Ghaedi, H. Salehi, A. Dolatshahi-Pirouz, A. Arpanaei, Incorporation of mesoporous silica nanoparticles into random electrospun PLGA and PLGA/gelatin nanofibrous scaffolds enhances mechanical and cell proliferation properties, Materials Science \& Engineering C 66 (2016) 25-32.

[34] H. Lee, S.M. Dellatore, W.M. Miller, P.B. Messersmith, Mussel-inspired surface chemistry for multifunctional coatings, Science (New York, N.Y.) 318(5849) (2007) 426-430.

[35] Z. Xu, N. Wang, P. Liu, Y. Sun, Y. Wang, F. Fei, S. Zhang, J. Zheng, B. Han, Poly(Dopamine) Coating on 3D-Printed Poly-Lactic-Co-Glycolic Acid/ $\beta$-Tricalcium Phosphate Scaffolds for Bone Tissue Engineering, Molecules (Basel, Switzerland) 24(23) (2019).

[36] S. Zhao, M. Golestani, A. Penesyan, B. Deng, C. Zheng, V. Strezov, Antibiotic enhanced dopamine polymerization for engineering antifouling and antimicrobial membranes, Chinese Chemical Letters (2019).

[37] Q. Huang, M. Liu, J. Chen, Q. Wan, J. Tian, L. Huang, R. Jiang, F. Deng, Y. Wen, X. Zhang, Y. Wei, Marrying the mussel inspired chemistry and KabachnikFields reaction for preparation of $\mathrm{SiO} 2$ polymer composites and enhancement removal of methylene blue, Applied Surface Science 422 (2017) 17-27.

[38] L. Peng, R. Guo, J. Lan, S. Jiang, S. Lin, Microwave-assisted deposition of silver nanoparticles on bamboo pulp fabric through dopamine functionalization, Applied Surface Science 386 (2016) 151-159.

[39] D. Shi, J. Shen, Z. Zhang, C. Shi, M. Chen, Y. Gu, Y. Liu, Preparation and properties of dopamine-modified alginate/chitosan-hydroxyapatite scaffolds with gradient structure for bone tissue engineering, Journal of Biomedical Materials Research Part A 107(8) (2019) 1615-1627. 
[40] J. Wu, L. Cao, Y. Liu, A. Zheng, D. Jiao, D. Zeng, X. Wang, D.L. Kaplan, X. Jiang, Functionalization of Silk Fibroin Electrospun Scaffolds via BMSC Affinity Peptide Grafting through Oxidative Self-Polymerization of Dopamine for Bone Regeneration, ACS applied materials \& interfaces 11(9) (2019) 8878-8895.

[41] Z.H. Lu, H. Owens, A Method to Improve the Quality of Silica Nanoparticles (SNPs) over Increasing Storage Durations, Journal of Nanoparticle Research 20(8) (2018).

[42] U.H.F. Bunz, Breath Figures as A Dynamic Templating Method for Polymers and Nanomaterials, Advanced Materials 18(8) (2006) 973-989.

[43] M. Srinivasarao, D. Collings, A. Philips, S. Patel, Three-Dimensionally Ordered Array of Air Bubbles in A Polymer Film, Science 292(5514) (2001) 79-83.

[44] J. Zheng, H. Zhang, Z. Zhao, C.C. Han, Construction of Hierarchical Structures by Electrospinning or Electrospraying, Polymer 53(2) (2011).

[45] K.A.G. Katsogiannis, G.T. Vladisavljevic, S. Georgiadou, Porous Electrospun Polycaprolactone (PCL) Fibres by Phase Separation, European Polymer Journal 69 (2015) 284-295.

[46] Z.H. Qi, H. Yu, Y.M. Chen, M.F. Zhu, Highly porous fibers prepared by electrospinning a ternary system of nonsolvent/solvent/poly(L-lactic acid), Materials Letters 63(3-4) (2009) 415-418.

[47] P. Pan, B. Zhu, W. Kai, T. Dong, Y. Inoue, Effect of Crystallization Temperature on Crystal Modifications and Crystallization Kinetics of Poly(L-lactide), Journal of Applied Polymer Science 107(1) (2008) 54-62.

[48] N. Naga, Y. Yoshida, M. Inui, K. Noguchi, S. Murase, Crystallization of Amorphous Poly(lactic acid) Induced by Organic Solvents, Journal of Applied Polymer Science 119(4) (2011) 2058-2064. 
[49] L. Haeshin, P.L. Bruce, B.M. Phillip, A reversible wet/dry adhesive inspired by mussels and geckos, Nature 448(7151) (2007) 338.

[50] A. Postma, Y. Yan, Y. Wang, A.N. Zelikin, E. Tjipto, F. Caruso, Selfpolymerization of dopamine as a versatile and robust technique to prepare polymer capsules, Chemistry of Materials 21(14) (2009) 3042-3044.

[51] J. Li, Y. Li, L. Li, A.F.T. Mak, F. Ko, L. Qin, Preparation and biodegradation of electrospun PLLA/keratin nonwoven fibrous membrane, Polymer Degradation and Stability 94(10) (2009) 1800-1807.

[52] Y. Lia, Y. Guoa, J. Gea, P.X. Mac, B. Lei, In situ silica nanoparticles-reinforced biodegradable poly(citrate-siloxane) hybrid elastomers with multifunctional properties for simultaneous bioimaging and bone tissue regeneration, Applied Materials Today 10 (2018) 153-163.

[53] C. Dhand, S.T. Ong, N. Dwivedi, S.M. Diaz, J.R. Venugopal, B. Navaneethan, M.H.U.T. Fazil, S. Liu, V. Seitz, E. Wintermantel, R.W. Beuerman, S. Ramakrishna, N.K. Verma, R. Lakshminarayanan, Bio-inspired in situ crosslinking and mineralization of electrospun collagen scaffolds for bone tissue engineering, Biomaterials 104 (2016) 323-338. 